\title{
Deep Inner-Shell Multiphoton Ionization by Intense X-Ray Free-Electron Laser Pulses
}

\author{
H. Fukuzawa, ${ }^{1,2}$ S.-K. Son, ${ }^{3}$ K. Motomura, ${ }^{1}$ S. Mondal, ${ }^{1}$ K. Nagaya, ${ }^{2,4}$ S. Wada, ${ }^{2,5}$ X.-J. Liu, ${ }^{6}$ R. Feifel, ${ }^{7}$ \\ T. Tachibana, ${ }^{1}$ Y. Ito, ${ }^{1}$ M. Kimura, ${ }^{1}$ T. Sakai, ${ }^{4}$ K. Matsunami, ${ }^{4}$ H. Hayashita, ${ }^{5}$ J. Kajikawa, ${ }^{5}$ P. Johnsson, ${ }^{8}$ \\ M. Siano, ${ }^{9}$ E. Kukk, ${ }^{10}$ B. Rudek, ${ }^{1,12}$ B. Erk, ${ }^{11,12}$ L. Foucar, ${ }^{1,13}$ E. Robert, ${ }^{6}$ C. Miron, ${ }^{6}$ K. Tono, ${ }^{14}$ \\ Y. Inubushi, ${ }^{2}$ T. Hatsui, ${ }^{2}$ M. Yabashi, ${ }^{2}$ M. Yao, ${ }^{4}$ R. Santra, ${ }^{3,15, *}$ and K. Ueda ${ }^{1,2, \dagger}$ \\ ${ }^{1}$ Institute of Multidisciplinary Research for Advanced Materials, Tohoku University, Sendai 980-8577, Japan \\ ${ }^{2}$ RIKEN SPring-8 Center, Kouto 1-1-1, Sayo, Hyogo 679-5148, Japan \\ ${ }^{3}$ Center for Free-Electron Laser Science (CFEL), DESY, 22607 Hamburg, Germany \\ ${ }^{4}$ Department of Physics, Kyoto University, Kyoto 606-8502, Japan \\ ${ }^{5}$ Department of Physical Science, Hiroshima University, Higashi-Hiroshima 739-8526, Japan \\ ${ }^{6}$ Synchrotron SOLEIL, L'Orme des Merisiers, Saint-Aubin, BP 48, FR-91192 Gif-sur-Yvette Cedex, France \\ ${ }^{7}$ Department of Physics and Astronomy, Uppsala University, P.O. Box 516, SE-751 20 Uppsala, Sweden \\ ${ }^{8}$ Department of Physics, Lund University, P.O. Box 118, 22100 Lund, Sweden \\ ${ }^{9}$ Blackett Laboratory, Imperial College London, London SW7 2AZ, United Kingdom \\ ${ }^{10}$ Department of Physics and Astronomy, University of Turku, 20014, Finland \\ ${ }^{11}$ Max-Planck Advanced Study Group at CFEL, 22607 Hamburg, Germany \\ ${ }^{12}$ Max-Planck-Insitut für Kernphysik, 69117 Heidelberg, Germany \\ ${ }^{13}$ Max-Planck-Insitut für medizinische Forschung, 69120 Heidelberg, Germany \\ ${ }^{14}$ Japan Synchrotron Radiation Research Institute (JASRI), Kouto 1-1-1, Sayo, Hyogo 679-5198, Japan \\ ${ }^{15}$ Department of Physics, University of Hamburg, 20355 Hamburg, Germany
}

(Received 23 September 2012; revised manuscript received 4 February 2013; published 26 April 2013)

We have investigated multiphoton multiple ionization dynamics of xenon atoms using a new x-ray free-electron laser facility, SPring-8 Angstrom Compact free electron LAser (SACLA) in Japan, and identified that $\mathrm{Xe}^{n+}$ with $n$ up to 26 is produced at a photon energy of $5.5 \mathrm{keV}$. The observed high charge states $(n \geq 24)$ are produced via five-photon absorption, evidencing the occurrence of multiphoton absorption involving deep inner shells. A newly developed theoretical model, which shows good agreement with the experiment, elucidates the complex pathways of sequential electronic decay cascades accessible in heavy atoms. The present study of heavy-atom ionization dynamics in high-intensity hard- $\mathrm{x}-$ ray pulses makes a step forward towards molecular structure determination with x-ray free-electron lasers.

PACS numbers: 32.80.Fb, 02.70.Uu, 32.80.Wr, 41.60.Cr

Multiphoton processes in the optical regime are wellknown phenomena investigated for decades. The advent of extreme ultraviolet [1,2] and $\mathrm{x}$-ray [3] free-electron lasers (FELs), with femtosecond pulse width, has led to renewed interest in multiphoton processes in the extreme ultraviolet to x-ray spectral region. Some of the recent works on atoms and small molecules were carried out at FLASH [1] in Germany [4-6] and at the SCSS test accelerator [2] in Japan [7-11], as well as at LCLS [3] in USA [12-19]. The motivation for these studies has been not only to reveal the pathways of the multiphoton multiple ionization newly opened by these light sources [4,10-13,20-24] but also to employ these processes as the basis for a new type of spectroscopy for chemical analysis $[17,18,25]$. These processes have attracted broad and intense attention in connection with electronic radiation damage [26] in x-ray imaging. So far, however, multiphoton experiments have been limited to the photon energy range up to $2 \mathrm{keV}$, i.e., the upper photon energy limit of the AMO beam line at LCLS. Multiphoton processes at higher photon energies are of particular importance because of their direct relevance for the electronic damage in high-resolution $\mathrm{x}$-ray imaging measurements.

Femtosecond crystallography with $\mathrm{x}$-ray free-electron lasers (XFELs) [27,28] is in the process of revolutionizing molecular structure determination, but the phase problem remains a major obstacle to structural reconstruction from measured x-ray scattering patterns. A promising approach to solving the phase problem in a rigorous way is highintensity multiwavelength anomalous diffraction [29], which makes use of heavy atoms as reference scatterers. This technique (and related techniques) requires the ability to describe the dynamical behavior of heavy atoms in highintensity hard-x-ray beams. Thus, the current experiment, which will be described below, is a fundamental test of our ability to provide such a description. The description is enormously challenging because of the complex dynamical pathways accessible in heavy atoms when using hard $\mathrm{x}$ rays. Therefore, with the current experiment and theory, we are taking a step towards addressing the phase problem in femtosecond crystallography using XFELs.

In March 2012, a new XFEL facility, the SPring-8 Angstrom Compact free electron LAser (SACLA) [30], 
started user operation in Japan. Using this new facility, we have investigated multiphoton multiple ionization dynamics of argon and xenon atoms in intense hard x-ray pulses. The Ar results are used to determine the peak fluence of the XFEL pulse. The Xe $L$-shell thresholds are around $5 \mathrm{keV}$. The intense $\mathrm{x}$-ray pulses from SACLA, with a photon energy above those deep inner-shell thresholds, induce complex multiple ionization dynamics characterized by the absorption of several photons. We identify that for a single Xe atom, absorption of 5 or more photons of $5.5 \mathrm{keV}$ induces emission of up to 26 electrons. As a consequence of intra-atomic electron-electron interactions, each photon causes the ejection of about 5 electrons on average.

From the pioneering work on the light neon atom, we learned that $\mathrm{x}$-ray multiphoton multiple ionization is well characterized by a sequence of one-photon ionizations accompanied by decay processes $[12,20]$. The complexity increases for heavy atoms, where one-photon ionization of a deep inner shell initiates a decay cascade, i.e., a series of decay steps leading to the emission of several electrons [31]. The extremely large number of $\mathrm{x}$-ray photons in an XFEL pulse is responsible for triggering further photoionization from deep inner shells during and after a decay cascade. Thus sequential multiphoton absorption involving deep inner shells becomes very complicated. As a consequence of its sequential nature, x-ray multiphoton absorption depends primarily on the fluence, which is the number of photons (or the pulse energy) per unit area, rather than the (peak) intensity $[12,19,20]$. The minimum fluence required for multiphoton absorption is estimated as follows. For example, the photoionization cross section of neutral Xe at $5.5 \mathrm{keV}$ is $\sim 0.166 \mathrm{Mb}$, so the fluence to saturate one-photon absorption is $\sim 6 \times 10^{10}$ photons $/ \mu \mathrm{m}^{2}$, which corresponds to $\sim 50 \mu \mathrm{J} / \mu \mathrm{m}^{2}$ for $5.5-\mathrm{keV}$ photons. If the fluence of an $\mathrm{x}$-ray pulse is close to or higher than this fluence, one expects that more than one photon will be absorbed by a xenon atom within one pulse.

The experiment has been carried out at the experimental hutch 3 (EH3) of the beam line 3 (BL3) of SACLA [30]. A Kirkpatrick-Baez (KB) mirror system is permanently installed at EH3 [32]. The XFEL beam is focused by the $\mathrm{KB}$ mirror system to a focal size of $\sim 1 \mu \mathrm{m}$ (FWHM) in diameter. The sample gas (argon or xenon) was introduced as a pulsed supersonic gas jet [33] to the focus point of the XFEL pulses, in the ultrahigh-vacuum reaction chamber. The source volume of the ions was roughly a cylindrical shape of $\sim 1 \mu \mathrm{m}$ in diameter and $\sim 2 \mathrm{~mm}$ (i.e., the diameter of the molecular beam) along the XFEL beam. The photon energy was set at $5.5 \mathrm{keV}$. The photon band width was $\sim 60 \mathrm{eV}$ (FWHM). The repetition rate of the XFEL pulses was $10 \mathrm{~Hz}$. The pulse length has not been measured, but it was estimated to be in the range between 10 and $30 \mathrm{fs}$ (FWHM) [34]. XFEL pulse energies were measured by the beam-position monitor [35] located upstream of the beam line. The monitor was calibrated by a calorimeter [36] so that output signals from the monitor could be transformed to the absolute value of the pulse energy. The measured value was $239 \mu \mathrm{J}$ per pulse on average. The relative $\mathrm{x}$-ray pulse energy passing through the interaction point was also measured shot by shot by a $p$-intrinsic- $n$ photodiode. The shot-by-shot pulse energy fluctuation was $\pm 25 \%$ ( $50 \%$ FWHM).

Ions produced in the source volume described above were extracted towards the ion time-of-flight (TOF) spectrometer [37] equipped with microchannel plates and a delay-line anode (Roentdek HEX80) [38]. Signals from delay-line anode and microchannel plates were recorded by the digitizer and analyzed by a software discriminator [39]. The arrival time and the arrival position of each ion were determined. The position information was used to compensate the TOF that varied depending on the departure positions perpendicular to spectrometer axis. Figure 1 depicts the TOF spectrum for xenon ions after compensation of the TOF. We can clearly see ions with a charge state of up to +26 and well-resolved isotopes at different charge states.

Let us now describe our theoretical approach to x-ray multiphoton multiple ionization dynamics. We employ the XATOM toolkit [23], based on the rate equation approach [20] and the Hartree-Fock-Slater method. For Xe deep inner-shell ionization, the straightforward sequential ionization model becomes tremendously challenging, because (a) there are too many electronic configurations involving multiple holes and too many atomic data (photoionization cross sections, Auger and Coster-Kronig rates, and fluorescence rates), and (b) the matrix size for the set of coupled rate equations is too large to be solved directly. We have addressed the latter by introducing a Monte Carlo approach in Refs. [19,24] for Xe $M$-shell ionization

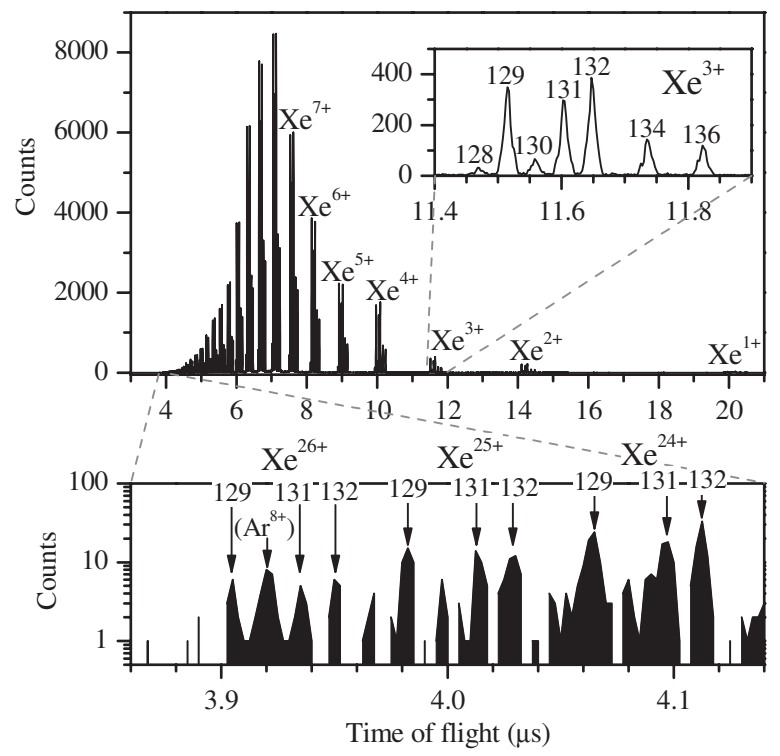

FIG. 1. The ion time-of-flight spectrum of Xe recorded at the photon energy of $5.5 \mathrm{keV}$ at the full XFEL pulse energy. 


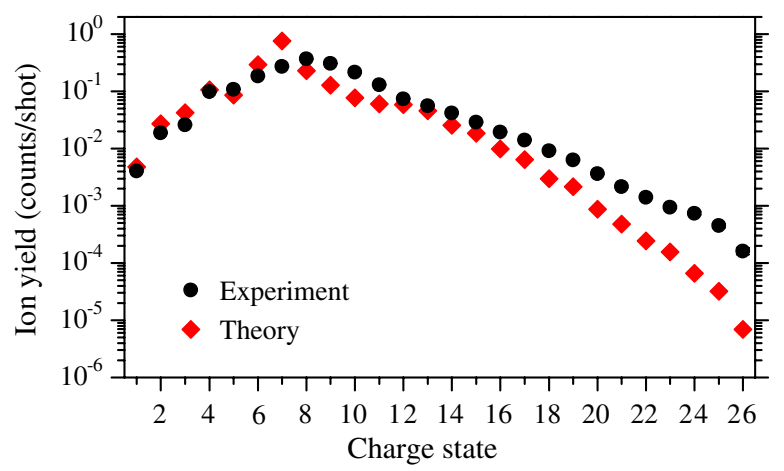

FIG. 2 (color online). Experimental and theoretical charge state distributions of $\mathrm{Xe}$ at the photon energy of $5.5 \mathrm{keV}$ with the peak fluence of $47 \mu \mathrm{J} / \mu \mathrm{m}^{2}$.

dynamics with precalculated atomic data. For Xe $L$-shell ionization dynamics considered here, however, the complexity is further increased: $\sim 2 \times 10^{7}$ coupled rate equations with $\sim 2 \times 10^{9}$ atomic data need to be solved, so that it becomes impractical to precalculate all the atomic data required for the rate equations. Therefore, we have extended XАTOM by applying the Monte Carlo procedure for both calculating atomic data and searching probable ionization pathways. This extension enables us to treat ionization dynamics of heavy atoms with no limit of configurational space. All calculated ion yields presented here are obtained by three-dimensional integration [19] over the interaction volume according to the instrumental configuration.

In order to compare the theoretical results with the experimental results, we need the peak fluence of the XFEL pulse employed for our experiment. The fluence at the center of the X-ray beam focus defines the peak fluence $F_{\text {peak }}$, which is given by

$$
F_{\text {peak }}=\frac{4 \ln 2}{\pi} \times \frac{E}{A} \times T,
$$

where $E$ is the nominal pulse energy from the monitor, $T$ is the transmission, $A$ is the focal area given in FWHM $\times$ FWHM, and the coefficient of $4 \ln 2 / \pi$ comes from the assumed Gaussian focal shape. In order to determine the peak fluence of the XFEL pulse, we have measured the charge state distribution of Ar atoms and compared it with our benchmark calculations [40].

Figure 2 depicts the charge state distribution of $\mathrm{Xe}$ at the peak fluence of $47 \mu \mathrm{J} / \mu \mathrm{m}^{2}$. The charge state distribution varies as the peak fluence varies (not shown here). We also compare the theoretical charge state distributions at this peak fluence with experiment. The photon energy of $5.5 \mathrm{keV}$ is above the $L$-shell threshold for charge states up to +23 according to our calculations. Thus, direct two-photon ionization [13] barely contributes to charge formation because not only is its cross section very small but also one-photon ionization is always available up to

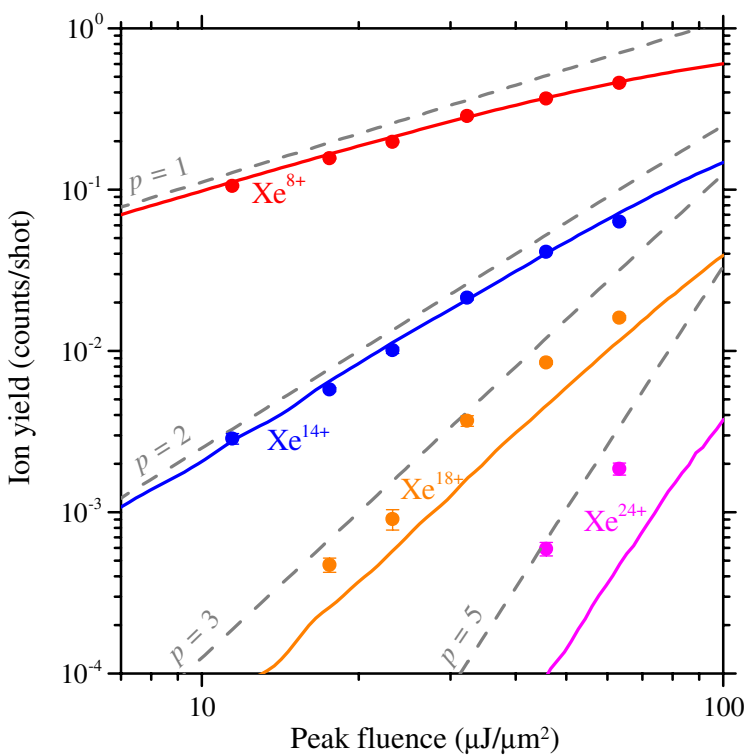

FIG. 3 (color online). XFEL fluence dependence of the ion yields for $\mathrm{Xe}^{n+}(n=8,14,18$, and 24). Closed circles with the error bars depict the experimental results and solid lines depict the theoretical results. Lines with slope $p=1,2,3$, and 5 are also shown as broken lines to guide the eye. The uncertainty of the peak fluence is expected to be $\sim \pm 10 \%$.

+23 . There is no apparent signature of resonance-enabled ionization enhancement [19] because the fluence is not enough to form high charge states that involve resonance excitation. The discrepancy between theory and experiment may be attributed to the nonrelativistic treatment and lack of shakeoff in the current theoretical model. The shakeoff process can further ionize valence electrons after photoionization, and some decay channels might be absent without relativity [24]. Inclusion of both relativity and shakeoff tends to produce higher charge states. In spite of these limitations, the experimental and theoretical results are in reasonable agreement, at least semiquantitatively.

To obtain the fluence dependence of the yields for the xenon ions at different charge states in a pulse energy range wider than the shot-by-shot pulse energy fluctuation, we recorded the spectra not only at the full XFEL pulse energy but also at a pulse energy attenuated by an aluminum foil of $25 \mu \mathrm{m}$ thickness located upstream of the KB mirror system. The attenuated pulse energy was $38 \%$ of the full pulse energy according to the reading of the $p$-intrinsic- $n$ photodiode. In Fig. 3, the ion yields for $\mathrm{Xe}^{n+}(n=8,14$, 18 , and 24) are plotted as a function of the peak fluence. To obtain several data points with respect to the peak fluence, we first merged the results measured at two different pulse energies, and then rebinned the data. Straight lines with a slope of 1,2,3, and 5 are also shown as a guide to the eye. All theoretical ion yields are scaled by a single factor such that the yield of $\mathrm{Xe}^{8+}$ at $47 \mu \mathrm{J} / \mu \mathrm{m}^{2}$ is matched with that in the experiment. The yield of $\mathrm{Xe}^{8+}$ exhibits a slope of less than one, illustrating that this ion is produced 


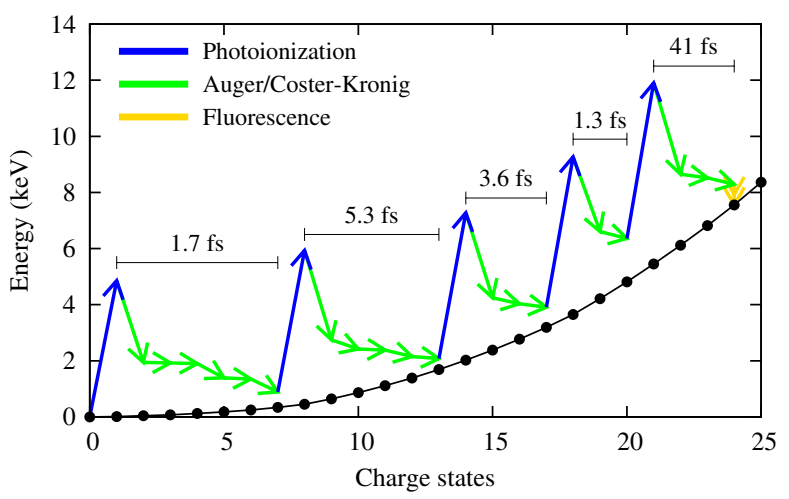

FIG. 4 (color online). An exemplary pathway of multiphoton multiple ionization of $\mathrm{Xe}$ at $5.5 \mathrm{keV}$. The black solid line with dots indicates the ground-configuration energy for given charge states, and the energy of neutral Xe is set to zero.

by single-photon absorption and that the single-photon absorption process is close to saturation. Before reaching the saturation point, $\sim 50 \mu \mathrm{J} / \mu \mathrm{m}^{2}$ as estimated earlier, the yield of $\mathrm{Xe}^{14+}$ and $\mathrm{Xe}^{18+}$ exhibits a slope close to two and three, respectively. These slopes before saturation directly suggest the number of absorbed photons to yield the corresponding charge states. The theoretical results (solid lines) reproduce very well the fluence dependence of the individual charge states. At higher fluences, the yields start to bend over due to the saturation effect, as clearly shown by the solid lines. For $\mathrm{Xe}^{24+}$, it is hard to fit the experimental data at low fluences; according to our calculations, $\mathrm{Xe}^{24+}$ is produced via five-photon absorption. The number of absorbed photons may be overestimated by at most one due to the limitations of the current model.

Figure 4 depicts one typical pathway yielding $\mathrm{Xe}^{24+}$. The plot illustrates that the total energy of the system varies in the course of the ionization steps. After $L$-shell photoionization (blue arrows), the energetically excited core-hole state relaxes via a series of Auger and Coster-Kronig decays (green arrows), and/or fluorescences (yellow arrows). Note that another photoionization occurs before the atom fully relaxes to the ground configuration. We find it useful to view the multiphoton multiple ionization dynamics occurring in a single atom in terms of quantum evaporation of electrons: $\mathrm{x}$ rays heat up the atomic system to highly excited states, and then the system relaxes primarily by emitting electrons with characteristic energies. The excess energy is shared among the electrons via electron-electron collisions, resulting in the ejection of 24 electrons in total.

For each photoionization, the deep single-core vacancy is rapidly filled up within sub-fs and the time scale of the accompanying Auger and Coster-Kronig decay cascade ranges from a few fs to tens of fs, as indicated in Fig. 4. The time scale of fluorescences at the highest charge state is about $0.7 \mathrm{ps}$. In the present calculations, the pulse shape is assumed to be a Gaussian of 30 fs (FWHM). In the regime of sequential multiphoton absorption, the results are not sensitive to the pulse shape or spikiness of individual pulses [20], unless the pulse duration is much shorter than the decay time scale. For example, at a fluence of $60 \mu \mathrm{J} / \mu \mathrm{m}^{2}$, we obtain the same mean charge $(+6.2)$ for pulse durations greater than $\sim 5$ fs (FWHM). The mean charge is reduced to +5.9 for a pulse duration of 1 fs (FWHM). The frustrated absorption [12,14], which would be expected to play a substantial role here if the pulse duration came close to the single-core-hole lifetime (sub-fs for Xe $L$-shell vacancy), is irrelevant for the pulse durations currently available at sufficiently high $\mathrm{x}$-ray fluence.

In summary, we have investigated multiphoton multiple ionization dynamics of the xenon atom using the new XFEL facility SACLA as well as a newly developed theoretical model, demonstrating the occurrence of multiphoton absorption by a single atom in the $\mathrm{x}$-ray regime above $5 \mathrm{keV}$ for the first time. The absolute fluence of the XFEL pulses has been determined using two-photon processes in argon atoms with the help of benchmark $a b$ initio calculations. The hard-x-ray photon energy and the heavy atoms are particularly relevant to the emerging area of molecular structure determination with XFELs, promising to solve the phase problem. In contrast to previous XFEL experiments at lower photon energies, frustrated absorption $[12,14]$ and resonance-enabled ionization enhancement [19] do not appear to be important with current XFEL parameters in the hard x-ray regime. Understanding of ionization dynamics of heavy atoms exposed to highintensity hard-x-ray beams provides useful input for future molecular imaging experiments using XFELs.

The experiments were performed at SACLA with the approval of JASRI and the program review committee (No. 2012A8036). This study was supported by the X-ray Free Electron Laser Utilization Research Project and the X-ray Free Electron Laser Priority Strategy Program of the Ministry of Education, Culture, Sports, Science and Technology of Japan (MEXT), by the Japan Society for the Promotion of Science (JSPS) KAKENHI Grants No. 21244062, No. 23241033, by MEXT KAKENHI Grant No. 22740264, by the Proposal Program of SACLA Experimental Instruments of RIKEN and by the IMRAM project. S. M. acknowledges financial support from JSPS. R. F. acknowledges financial support from the Swedish Research Council (VR). P. J. acknowledges support from the Swedish Research Council (VR) and the Swedish Foundation for Strategic Research (SSF).

*robin.santra@cfel.de †ueda@tagen.tohoku.ac.jp

[1] W. Ackermann et al., Nat. Photonics 1, 336 (2007).

[2] T. Shintake et al., Nat. Photonics 2, 555 (2008).

[3] P. Emma et al., Nat. Photonics 4, 641 (2010). 
[4] M. Richter, M. Ya. Amusia, S. V. Bobashev, T. Feigl, P. N. Juranić, M. Martins, A. A. Sorokin, and K. Tiedtke, Phys. Rev. Lett. 102, 163002 (2009).

[5] M. Kurka et al., New J. Phys. 12, 073035 (2010).

[6] A. Rouzee et al., Phys. Rev. A 83, 031401(R) (2011).

[7] K. Motomura et al., J. Phys. B 42, 221003 (2009).

[8] A. Yamada et al., J. Chem. Phys. 132, 204305 (2010).

[9] T. Sato et al., J. Phys. B 44, 161001 (2011).

[10] E. V. Gryzlova et al., Phys. Rev. A 84, 063405 (2011).

[11] A. Hishikawa et al., Phys. Rev. Lett. 107, 243003 (2011).

[12] L. Young et al., Nature (London) 466, 56 (2010).

[13] G. Doumy et al., Phys. Rev. Lett. 106, 083002 (2011).

[14] M. Hoener et al., Phys. Rev. Lett. 104, 253002 (2010).

[15] J. P. Cryan et al., Phys. Rev. Lett. 105, 083004 (2010).

[16] L. Fang et al., Phys. Rev. Lett. 105, 083005 (2010).

[17] N. Berrah et al., Proc. Natl. Acad. Sci. U.S.A. 108, 16912 (2011).

[18] P. Salèn et al., Phys. Rev. Lett. 108, 153003 (2012).

[19] B. Rudek et al., Nat. Photonics 6, 858 (2012).

[20] N. Rohringer and R. Santra, Phys. Rev. A 76, 033416 (2007).

[21] M. G. Makris, P. Lambropoulos, and A. Mihelic, Phys. Rev. Lett. 102, 033002 (2009).

[22] P. Lambropoulos, G. M. Nikolopoulos, and K. G. Papamihail, Phys. Rev. A 83, 021407(R) (2011).

[23] S.-K. Son, L. Young, and R. Santra, Phys. Rev. A 83, 033402 (2011).
[24] S.-K. Son and R. Santra, Phys. Rev. A 85, 063415 (2012).

[25] R. Santra, N. V. Kryzhevoi, and L. S. Cederbaum, Phys. Rev. Lett. 103, 013002 (2009).

[26] H. M. Quiney and K. A. Nugent, Nat. Phys. 7, 142 (2011).

[27] H. N. Chapman et al., Nature (London) 470, 73 (2011).

[28] L. Redecke, K. Nass et al., Science 339, 227 (2013).

[29] S.-K. Son, H. N. Chapman, and R. Santra, Phys. Rev. Lett. 107, 218102 (2011).

[30] T. Ishikawa et al., Nat. Photonics 6, 540 (2012).

[31] T. A. Carlson, W. E. Hunt, and M. O. Krause, Phys. Rev. 151, 41 (1966).

[32] H. Yumoto et al., Nat. Photonics 7, 43 (2013).

[33] K. Nagaya et al., J. Electron Spectrosc. Relat. Phenom. 181, 125 (2010).

[34] Y. Inubushi et al., Phys. Rev. Lett. 109, 144801 (2012).

[35] K. Tono, T. Kudo, M. Yabashi, T. Tachibana, Y. Feng, D. Fritz, J. Hasting, and T. Ishikawa, Rev. Sci. Instrum. 82, 023108 (2011).

[36] M. Kato et al., Appl. Phys. Lett. 101, 023503 (2012).

[37] X.-J. Liu et al., Rev. Sci. Instrum. 80, 053105 (2009).

[38] O. Jagutzki et al., IEEE Trans. Nucl. Sci. 49, 2477 (2002).

[39] K. Motomura et al., Nucl. Instrum. Methods Phys. Res., Sect. A 606, 770 (2009).

[40] See Supplemental Material at http://link.aps.org/ supplemental/10.1103/PhysRevLett.110.173005 for the Ar calibration. 